\title{
A Comparative Survey of the Iron-Sulphur Centres in the Cytoplasmic Membranes of Pseudomonas cichorii and Pseudomonas aptata
}

\author{
By DAVIDE ZANNONI ${ }^{1}$ AND W. JOHN INGLEDEW ${ }^{2 *}$ \\ ${ }^{1}$ Institute of Botany, University of Bologna, Bologna, Italy \\ ${ }^{2}$ Department of Biochemistry and Microbiology, University of St Andrews, St Andrews, UK
}

(Received 2 December 1983; revised 6 February 1984)

The respiratory chains of the related phytopathogenic bacteria Pseudomonas cichorii and Pseudomonas aptata were investigated using the technique of electron paramagnetic resonance which readily detects iron-sulphur centres. In the cytoplasmic membranes of both bacteria three ferredoxin-like centres and a centre detected in the oxidized state $(g=2.015)$ were resolved. In addition, a Rieske-type iron-sulphur centre $\left(g_{\mathrm{y}}=1.90\right)$ was observed in $P$. cichorii but not in $P$. aptata. The absence of the Rieske centre in $P$. aptata parallels the absence of cytochromes $c$ in this bacterium.

\section{INTRODUCTION}

Pseudomonas cichorii and Pseudomonas aptata are fluorescent phytopathogenic bacteria. A study of their membrane-bound cytochromes has revealed that a linear respiratory chain is functional in $P$. aptata, cytochromes $c$ being absent and the terminal oxidase being an autooxidizable cytochrome $b$ (called cytochrome $o$ ). In contrast, $P$. cichorii appears to retain a similar cytochrome $o$ pathway but has in addition a second pathway involving cytochromes $c$ to a distinct auto-oxidizable cytochrome $b$. These cytochromes $b$ differ in their mid-point oxidationreduction potentials and their sensitivities to cyanide (Zannoni, 1982).

In this paper we have applied the techniques of electron paramagnetic resonance spectroscopy (EPR) to the study of these respiratory chains.

\section{METHODS}

Cell growth, harvesting, cell breakage and the preparation of cytoplasmic membrane fragments. Pseudomonas cichorii (NCPPB 907) and P. aptata (NCPPB 2664) were grown in a medium of the following composition: D-glucose, $20 \mathrm{~g}$; yeast extract (Difco), $5 \mathrm{~g} ; \mathrm{MgSO}_{4} .7 \mathrm{H}_{2} \mathrm{O}, 0.5 \mathrm{~g} ; \mathrm{KH}_{2} \mathrm{PO}_{4}, 0.6 \mathrm{~g} ; \mathrm{K}_{2} \mathrm{HPO}_{4}, 1 \mathrm{~g}$. The medium was adjusted to pH 7.2 in 1 litre of distilled water. Growth medium (10 l) supplemented with $0.5 \mathrm{ml}$ antifoam $\mathrm{C}$ emulsion was inoculated with $200 \mathrm{ml}$ of a growing culture (initial absorbance 0.05 at $660 \mathrm{~nm}$ ). The cells were harvested after growth for $18 \mathrm{~h}$ (final absorbance of about $1 \cdot 2$ ) at $30^{\circ} \mathrm{C}$ in a Microferm Fermentor (New Brunswick Scientific Co.), and then stirred at 250 r.p.m. and bubbled with humidified air at $61 \mathrm{~min}^{-1}$. The harvested cells were broken by French pressure cell treatment and the membrane fraction was separated by differential centrifugation as previously described (Zannoni, 1982).

Electron paramagnetic resonance spectroscopy. EPR spectra were obtained using a Bruker $200 \mathrm{D}$ EPR spectrometer (Bruker Analyt. Messtechnik, Forchleim, FRG) equipped with a cryostat (Oxford Instruments, Oxford, UK) and a liquid helium transfer system. The temperature was measured with a thermocouple.

Determination of oxidation-reduction mid-point potentials and other assays. Oxidation-reduction titrations were performed as described by Dutton (1971). The membrane preparation was suspended, to $20 \mathrm{mg}$ protein $\mathrm{ml}^{-1}$, in $50 \mathrm{~mm}$-TES buffer $\mathrm{pH} \mathrm{7.0.} \mathrm{The} \mathrm{following} \mathrm{mediators} \mathrm{were} \mathrm{used} \mathrm{at} \mathrm{between} 20$ and $50 \mu \mathrm{m}$ : dibromophenol indophenol ; 2-hydroxy-1,4-napthoquinone; 1,4-napthoquinone; duroquinone; indigo-disulphonate ; indigo-tetrasulphonate; 2,3,5,6-tetramethylphenylenediamine; $N$-methylphenazonium methosulphonate; methylviologen; 
benzylviologen; phenosafranine; anthraquinone-2-sulphonate; 2-hydroxy-napthoquinone. The samples were stored in liquid nitrogen until use. Protein concentrations were estimated by a modification of the Lowry method that included SDS.

\section{RESULTS}

In this paper the term ferredoxin is used to indicate an iron-sulphur centre which is EPRdetectable in its reduced form. The term HiPIP (high potential iron protein) is used for a centre which is EPR-detectable in its oxidized form. The EPR spectra shown are the first derivatives of the absorption spectra. This is standard practice with EPR and it gives much better resolution than the absorbance spectrum. A ferredoxin has either rhombic or axial geometry: the former will lead to a three-line first-derivative spectrum ( $g_{\mathrm{z}}$ peak, $g_{\mathrm{y}}$ peak and trough, $g_{\mathrm{x}}$ trough) and the latter to a two-line spectrum ( $g_{\|}$peak, $g_{\perp}$ peak and trough, i.e. $g_{\mathrm{x}}$ and $g_{\mathrm{y}}$ are superimposed). Each iron-sulphur centre will have its own three (or two) characteristic $g$ values [calculated from $g=v / \beta \mathrm{H}$, where $\beta$ is a constant (the Bohr magneton), $v$ the microwave frequency and $\mathrm{H}$ the magnetic field strength at the position of the line]. Spectra were taken at different temperatures because each centre has its own optimum temperature for observation (determined by its mechanisms and rates of relaxation and also by the incident microwave power) above and below which the signal intensity lessens (broadening and power saturation, respectively).

\section{Electron paramagnetic resonance spectra of $P$. aptata}

EPR spectra of $P$. aptata membranes either oxidized, NADH-reduced (anaerobic) or dithionite-reduced, are shown in Fig. 1 and the corresponding spectra of $P$. cichorii are shown in Fig. 2. In the spectrum of dithionite-reduced membranes obtained at $8 \mathrm{~K}$, two centres, distinguishable by their $g$ values and spectral lines shapes, could be resolved. Monitoring the various peaks and troughs from redox titrations and over a range of temperatures (not shown) led to the assignment of the $g=2.046$ peak and the $g=1.89$ trough to the same centre as $g_{z}$ and $g_{\mathrm{xy}}$, respectively (some rhombic distortion is suggested by the lineshape around $g=1.89$ ). The line shape of this centre has relatively little spectral overlap from other centres in the NADHreduced sample (Fig. $1 a$ ). In the NADH-reduced $8 \mathrm{~K}$ spectrum there was a centre with a $g_{\mathrm{z}}$ at 2.02 and $a g_{x y}$ in the region of 1.93 , but not so large as in the dithionite-reduced spectrum.

When spectra obtained at the higher temperature of $22 \mathrm{~K}$ were examined (Fig. $1 b$ ) it was observed that: (a) the $g=2.046 / 1.89$ centre was not present; (b) the rhombicity of the $g=$ $2.02 / 1.93$ centre could be seen as it was not free from overlap with the $g=2.046 / 1.89$ centre; (c) the line shape around the $g=1.93$ centre was different when comparing the dithionitereduced sample with the NADH-reduced sample and (d) the ratio of the signal amplitudes of the $\mathrm{NADH}$ and dithionite samples at $g=1.93$ was very different at $22 \mathrm{~K}$ compared to $8 \mathrm{~K}$. Points (c) and (d) suggest the presence of two $g=2.01 / 1.93$ centres, one being substrate-reducible (NADH), best observed at the higher temperatures and heavily saturated at $8 \mathrm{~K}$ (microwave power in Fig. 1 is $22 \mathrm{~mW}$ ), and the other not being NADH-reducible but dithionite-reducible and best observed at low temperatures. Studies of temperature dependence of the respective spectra and the analysis of redox titrations (Fig. 3), which resolved the $g=2.02 / 1.93$ centre into two components, confirmed this suggestion. Behaviour such as this, with iron-sulphur centres of this type, is well documented in succinate dehydrogenases and fumarate reductases (see Discussion).

In the oxidized sample at $8 \mathrm{~K}$ a large EPR signal with a peak at $g=2.015$ was observed. This centre was similar to centres described in succinate dehydrogenases, fumarate reductases and some other enzymes such as aconitase, and was due to a four or three iron sulphide cluster which is paramagnetic (EPR-detectable) in its physiologically oxidized form (designated HiPIP). Quantification of this HiPIP centre indicated that it was present at a similar concentration to the $g=2.02 / 1.93$ centres, which are also typical of succinate dehydrogenase. Measured under non-saturating conditions, HiPIP gave $0.13 \mathrm{nmol}(\mathrm{mg} \text { protein })^{-1}$; the $g=2.02 / 1.93$ dithionite- 


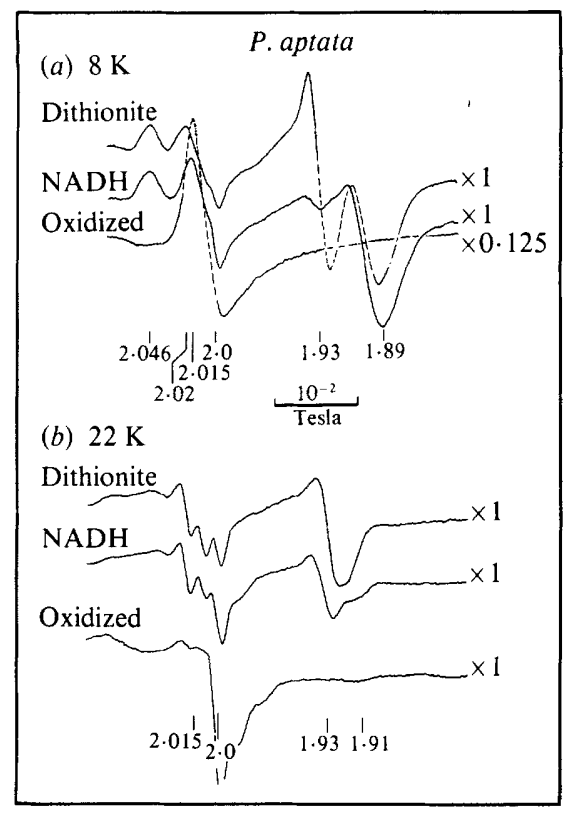

Fig. 1

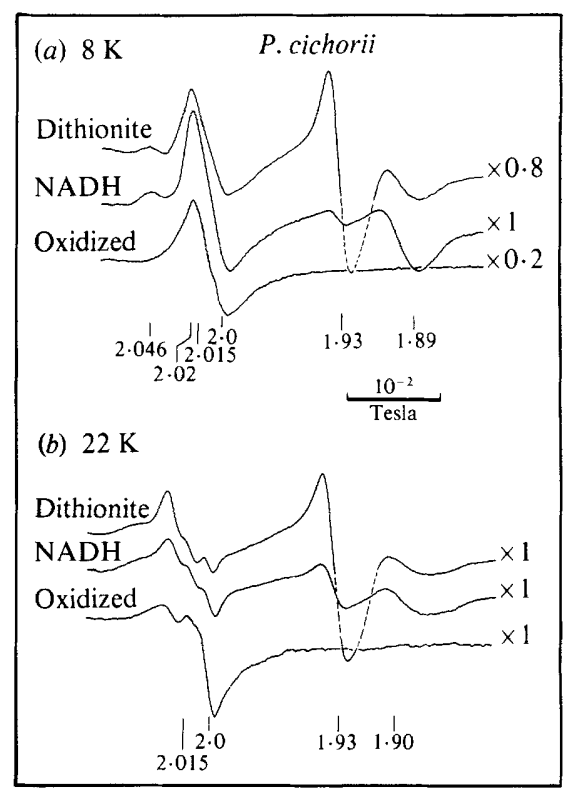

Fig. 2

Fig. 1. Electron paramagnetic resonance spectra of cytoplasmic membranes of $P$. aptata. Membrane samples were dithionite-reduced, NADH-reduced or oxidized. Spectra were taken at $(a) 8 \mathrm{~K}$ and $(b)$ $22 \mathrm{~K}$. The numbers at the bottom of the figures are $g$ values. The concentration of proteins was $40 \mathrm{mg} \mathrm{ml}^{-1}$. The EPR conditions were: microwave power, $22 \mathrm{~mW}$; modulation amplitude, $10^{-3}$ tesla; microwave frequency, $9.49 \mathrm{GHz}$; modulation frequency, $100 \mathrm{KHz}$.

Fig. 2. Electron paramagnetic resonance spectra of cytoplasmic membranes of $P$. cichorii. Spectra were taken at $(a) 8 \mathrm{~K}$ and $(b) 22 \mathrm{~K}$. Membrane samples were dithionite-reduced, NADH-reduced or oxidized. The numbers at the bottom of the figures are $g$ values. Other conditions were as for Fig. 1 .

reduced centres gave $0.15 \mathrm{nmol}(\mathrm{mg} \text { protein })^{-1}$ and the NADH-reduced centres gave $0.13 \mathrm{nmol}$ (mg protein) $)^{-1}$; and the $g=2.046 / 1.89$ centre gave $0.06 \mathrm{nmol}(\mathrm{mg} \text { protein })^{-1}$.

Oxidation-reduction titrations were performed to determine mid-point potentials (Fig. 3). The HiPIP centre gave a mid-point potential (pH 7.0) of approximately $+100 \mathrm{mV}$ and the $g=2.046 / 1.89$ ferredoxin a potential of $-210 \mathrm{mV}$. The $g=2.02 / 1.93$ ferredoxin titrated biphasically, suggesting two components (but see Discussion) with mid-point potentials at $-60 \mathrm{mV}$ and $-230 \mathrm{mV}$.

\section{EPR spectra of $P$. cichorii}

Figure 2 shows EPR spectra of oxidized and dithionite- or NADH-reduced $P$. cichorii membranes. The spectra were in some respects quite similar to those of P. aptata. The $g=2.046 / 1.89$ centre was present at the same concentration as in $P$. aptata at $0.06 \mathrm{nmol}(\mathrm{mg}$ protein) ${ }^{-1}$, although the $g=2.02 / 1.93$ centres (again resolvable into two components) were present at a higher concentration [dithionite-reduced, $0.38 \mathrm{nmol}(\mathrm{mg} \text { protein })^{-1}$ ]. In $P$. cichorii membranes an additional centre characterized by a $g_{\mathrm{y}}=1.90$ was seen at $22 \mathrm{~K}$. This resonance was similar to those of the Rieske iron-sulphur centres observed in other respiratory and photosynthetic electron transport chains in terms of its mid-point potential $(+200 \mathrm{mV}$, Fig. 3$), g$ values and temperature dependence (Rieske et al., 1964). This Rieske-like centre was clearly distinguishable from the $g=2.046 / 1.89$ centre on the basis of these same criteria. The Rieskelike centre also had a $g_{\mathrm{x}}$ resonance weakly indicated at $g=1 \cdot 78$; the expected $g_{\mathrm{z}}$ signal at $g=2.03$ could not be resolved because of overlapping signals. 

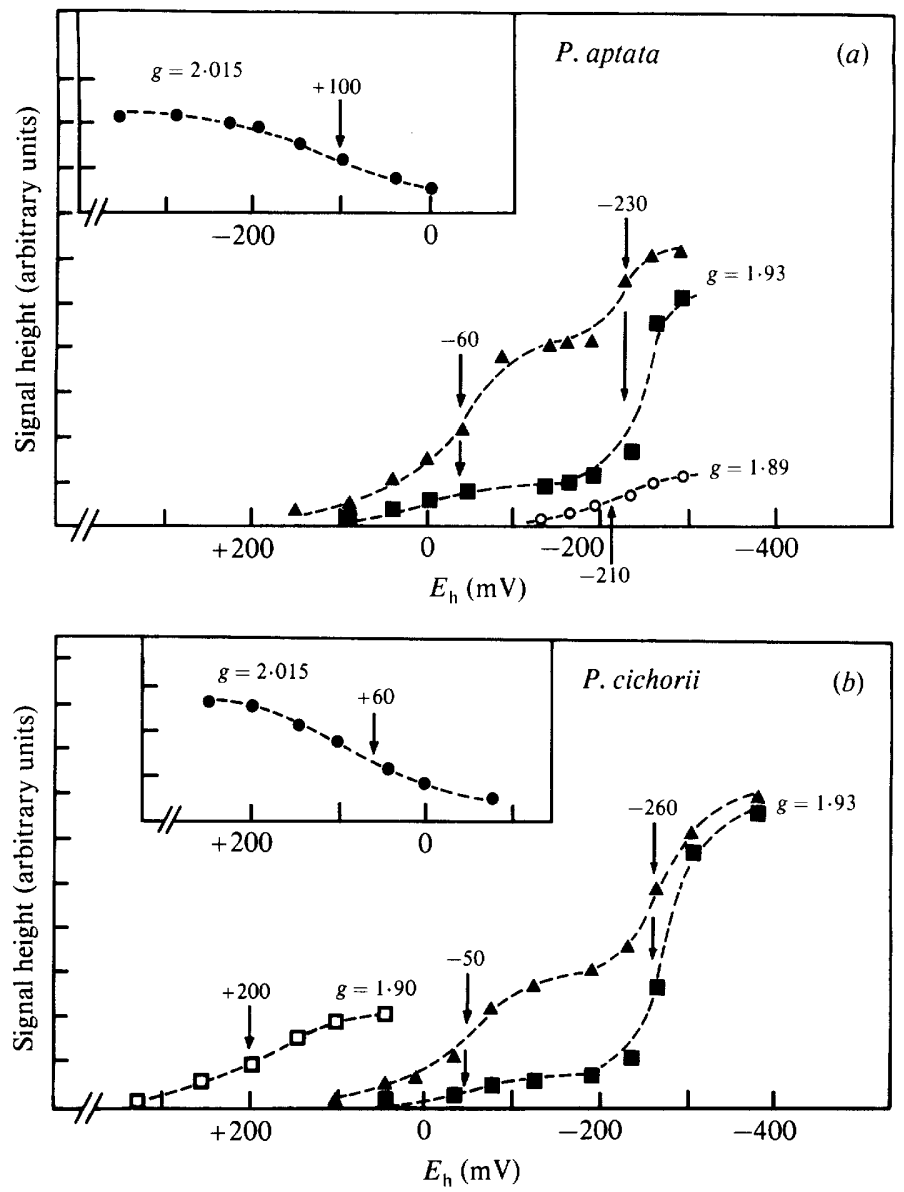

Fig. 3. Analysis of oxidation-reduction titrations of membrane fragments of (a) P. aptata and (b) $P$. cichorii. The curves are drawn for $n=1$ oxidation-reduction reactions. The EPR conditions were as for Fig. 1. The titration of the $g=1.93$ signal is shown from spectra taken at $8 \mathrm{~K}(\square)$ and $22 \mathrm{~K}(\boldsymbol{\Delta})$. The $g=1.89$ data $(O)$ are from $8 \mathrm{~K}$ spectra and are shown only for $P$. aptata (data are the same for $P$. cichorii but not shown). The Rieske data $(\square)$ were obtained from $22 \mathrm{~K}$ spectra of $P$. cichorii. The $g=2 \cdot 015$ data $(O)$ are from spectra at $8 \mathrm{~K}$.

\section{DISCUSSION}

Pseudomonas aptata and $P$. cichorii membranes contain a $g=2 \cdot 046 / 1.89$ ferredoxin with a mid-point potential of approximately $-210 \mathrm{mV}$ (Fig. 3, data not shown for $P$. cichorii), two $g=$ $2.02 / 1.93$ centres distinguishable by temperature dependence and mid-point potential $(-60 \mathrm{mV}$ and $-230 \mathrm{mV}$ for $P$. aptata and $-50 \mathrm{mV}$ and $-260 \mathrm{mV}$ for $P$. cichorii), and a HiPIP-type centre. A Rieske-type ferredoxin centre is present in $P$. cichorii but not in P. aptata.

Three of the iron-sulphur centres in each organism could be tentatively assigned to their succinate dehydrogenases by analogy with other systems. All known succinate dehydrogenases contain two $g=1.93$ centres and a HiPIP centre with mid-point potentials close to those reported here (Ohnishi et al., 1976; Ingledew \& Prince, 1977; Malkin \& Bearden, 1978; Ingledew et al., 1980; Beinert \& Albracht, 1982). An additional similarity between these centres and those of known succinate dehydrogenases is the difference in temperature dependence between the substrate-reducible $g=1.93$ centre (the higher potential centre) and the dithionite-reducible centre. Although in this paper the data are interpreted as indicating the presence of two $g=2.02 / 1.93$ ferredoxins, a more complex explanation exists in which only one 
centre is present. A change in the relaxation rate of the centre (hence temperature dependence) through titration of an adjacent but unknown species can give the appearance of two centres. However, this argument is beyond the scope of this paper (see Beinert \& Albracht, 1982).

The signal observed at $g=1.90$ at higher temperatures ( $22 \mathrm{~K}$ and above) and only found in $P$. cichorii is taken to be a Rieske-type centre by analogy with the centre detected in the ubiquinolcytochrome $c$ complex resolved from eukaryotic mitochondria by Rieske (Rieske et al., 1964). The $g_{\mathrm{y}}$ resonance is seen at $g=1.90$, the $g_{\mathrm{x}}$ resonance is weakly indicated at $g=1.78$ and the $g_{\mathrm{z}}$ resonance is expected at 2.03 but cannot be distinguished because of overlapping signals of unknown origin. The correlation between the presence of cytochromes $c$ in $P$. cichorii and the Rieske centre, compared to their absence in the closely related P. aptata, is of interest and suggests that in these pseudomonads cytochromes of the $c$ type and the Rieske centre are phenotypically and/or genetically linked. Such a correlation has been suggested before, but from comparison of widely different cell types (e.g. Escherichia coli and Paracoccus denitrificans; Ingledew et al., 1980). The observations reported in this paper strengthen the support for a combined function of cytochromes $c$ and Rieske centres.

D.Z. was the recipient of a short-term fellowship from the European Molecular Biology Organization. The support of the Science and Engineering Research Council of the UK is gratefully acknowledged.

\section{REFERENCES}

Beinert, H. \& Albracht, S. P. J. (1982). New insights, ideas and unanswered questions concerning iron-sulphur clusters in mitochondria. Biochimica et biophysica acta 683, 245-277.

DutTon, P. L. (1971). Oxidation-reduction potential dependence of the interaction of cytochromes, bacteriochlorophyll and carotenoids at $77 \mathrm{~K}$ in chromatophores of Chromatium D and Rhodopseudomonas aeruginosa. Biochemica et biophysica acta 226, 63-80.

Ingledew, W. J. \& Prince, R. C. (1977). Thermodynamic resolution of the iron sulphur centres of the succinic dehydrogenase of Rhodopseudomonas sphaeroides. Archives of Biochemistry and Biophysics 178, 303-307.

Ingledew, W. J., Reid, G. A., Poole, R. K., Blum, H. \& OHNishi, T. (1980). The iron sulphur centres of aerobically grown Escherichia coli K12. An electron paramagnetic resonance study. FEBS Letters 111, 223-227.
Malkin, R. \& Bearden, A. J. (1978). Membrane bound iron sulphur centres in photosynthetic systems. Biochimica et biophysica acta 505, 147-181.

OhNishi, T., Salerno, J. C., Winter, D. B., Lim, J., YU, C. A., Yu, L. \& King, T. E. (1976). Thermodynamic and EPR characteristics of two ferredoxintype iron-sulphur centres in the succinate-ubiquinone reductase segment of respiratory chain. Journal of Biological Chemistry 251, 2094-2104.

Rieske, J. S., ZAUG, W. S. \& HANSEN, R. E. (1964). Studies on the electron transfer system, LIX. Distribution of iron and of the component giving an electron paramagnetic resonance signal at $g=1.90$ in subfractions of complex III. Journal of Biological Chemistry 239, 3023-3036.

ZANNONI, D. (1982). Cytochrome $b$ type oxidases in the respiratory chains of the phytopathogenic fluorescent bacteria Pseudomonas cichorii and Pseudomonas aptata. Archives of Microbiology 133, 267-274. 